\title{
Effectiveness of Peer Support Interventions for Adolescents and Young Adults Living with HIV Globally
}

\author{
Helen Hernandez ${ }^{1}$, Hannah Craven ${ }^{1}$, Judith Toromo ${ }^{2}$, and Leslie A. Enane ${ }^{2}$
}

${ }^{1}$ Indiana University School of Medicine, ${ }^{2}$ The Ryan White Center for Pediatric Infectious Disease and Global Health, Department of Pediatrics, Indiana University School of Medicine

Background: Adolescents and young adults living with HIV (AYALHIV, ages 10-24) have poorer outcomes in care compared to other age groups. The global Treat All strategy aims to extend antiretroviral therapy (ART) to all individuals living with HIV. Efforts to improve outcomes in the AYALHIV population are essential to successfully achieving viral suppression and improving the long-term health outcomes of young people, and ultimately to ending the HIV pandemic. Peer support interventions are increasingly implemented by care programs, and are broadly favored by AYALHIV. This systematic review will evaluate the current evidence for quantitative effects of peer support interventions on AYALHIV treatment outcomes.

Methods: We searched MEDLINE, Embase, Global Health, CINAHL Complete, Cochrane, and Scopus databases for papers published between 1994 and October 2019, in all languages and regions. Studies of the effect of peer support interventions for AYALHIV on measures of adherence or retention in care were included. Solely qualitative studies and those without a comparator or control were excluded. Three researchers screened the papers independently, and those meeting criteria were included. Abstracted variables included study characteristics, participant population, peer intervention characteristics, and outcomes.

Results: The current evidence base for the effect of peer interventions for AYALHIV is very limited. Included studies were of small sample size, and primarily consisted of observational cohort studies with imperfect comparators. In initial analyses we find that studies of peer interventions have had mixed findings regarding the effect of such interventions on adherence to ART. By contrast, studies have consistently reported a favorable effect of peer interventions on increased retention in care among AYALHIV.

Conclusion/Implications: Rigorous studies of interventions to improve outcomes among AYALHIV are urgently needed. Further analyses in this review will examine differences according to study population, design, or intervention characteristics, which may underlie variable effects identified. 